\title{
INTRODUCTION \\ Advances and future directions in brain mapping in neurosurgery
}

\author{
Alexandra J. Golby, MD, ${ }^{1}$ Eric C. Leuthardt, MD, FNAI, ${ }^{2}$ Hugues Duffau, MD, PhD, ${ }^{3}$ and \\ Mitchel S. Berger, MD ${ }^{4}$
}

\begin{abstract}
'Department of Neurosurgery, Department of Radiology, Harvard Medical School, Brigham and Women's Hospital, Boston, Massachusetts; ${ }^{2}$ Department of Neurological Surgery, Division of Neurotechnology, Washington University, St. Louis, Missouri; ${ }^{3}$ Department of Neurosurgery, Gui de Chauliac Hospital, Montpellier University Medical Center, National Institute for Health and Medical Research (INSERM), U1051 Laboratory, Institute for Neurosciences of Montpellier, France; and ${ }^{2}$ Department of Neurological Surgery, Brain Tumor Research Center, University of California, San Francisco, California
\end{abstract}

$\mathrm{N}$ EUROSURGEONS have been directly involved with brain mapping efforts for well over a century. Direct access to the functioning human brain during surgery for intrinsic brain tumors, epilepsy, and functional neurosurgical procedures both requires individual functional brain mapping and presents an opportunity to precisely map human brain function. Several papers in this issue of Neurosurgical Focus extend our current understanding of cerebral organization with detailed examination of specific regions. Others introduce new technical nuances for intraoperative brain mapping. A third group of papers focuses on noninvasive brain mapping techniques that have emerged and been refined over the last several years.

We have organized this issue by technique. The first group of papers relates to direct cortical electrophysiology or optical techniques. Kramer et al. recorded from implanted electrodes in patients undergoing epilepsy surgery to determine the limits of frequency detection in order to develop a somatosensory brain-computer interface. Bander et al. propose a bipolar train-of-five paradigm for cortical motor mapping as an alternative to monopolar mapping. McDowell et al. describe a holistic protocol to prepare pediatric patients for awake surgery in order to maximize chances of success and minimize stress related to the experience. Oelschlägel et al. describe optical imaging for mapping motor and language function during awake surgery. Two papers explore white matter anatomy: Henderson et al. used tractography and the connectome to guide glioma surgery, whereas Fernández et al. provide an in-depth investigation of the white matter associated with Heschl's gyrus. Three papers focus on a variety of aspects related to functional MRI (fMRI): Catalino et al. review the use of resting fMRI for surgical planning, Nolan et al. studied a patient with a heterotopia, and Stopa et al. surveyed clinicians regarding their use of fMRI. Finally, two papers, one by Azad and Duffau and the other by Ellis et al., discuss mapping using noninvasive methods such as diffusion MRI and fMRI with comparison to the gold standard of intraoperative electrophysiology. The articles in this issue reveal the breadth of approaches that can now be brought to bear on the challenge of individual brain mapping while illustrating the numerous challenges that remain. We hope that Neurosurgical Focus readers will find these works informative and that these will inspire additional investigations.

https://thejns.org/doi/abs/10.3171/2019.11.FOCUS19880

\section{Disclosures}

Dr. Leuthardt reports the following conflicts of interest. Stock ownership: Neurolutions, Osteovantage, Inner Cosmos, Sora Imaging Solutions, Face to Face Biometrics, Caeli Vascular, and Immunovalent.

\section{Correspondence}

Alexandra J. Golby: agolby@bwh.harvard.edu. 